\title{
Multidisciplinary approach to a case of Lynch syndrome with colorectal, ovarian, and metastatic liver carcinomas
}

\author{
Masatoshi Shiono $\cdot$ Hideki Shimodaira $\cdot$ Mika Watanabe $\cdot$ Kei Takase $\cdot$ \\ Kiyoshi Ito · Koh Miura · Yuko Takami - Shoko Akiyama • Yuichi Kakudo • \\ Shin Takahashi $\cdot$ Masanobu Takahashi $\cdot$ Chikashi Ishioka
}

Received: 26 April 2012 / Accepted: 11 May 2012/Published online: 9 June 2012

(C) The Japan Society of Clinical Oncology 2012

\begin{abstract}
Lynch syndrome is an autosomal dominant disorder with an estimated prevalence of $3 \%$ of all colorectal cancers. It is attributed to germline mutations in DNA mismatch repair (MMR) genes, which confer increased susceptibility to cancers of the colorectum, endometrium, stomach, small intestine, hepatobiliary system, kidney, urinary bladder, brain, and ovary. We report a thought-provoking Lynch syndrome case with a family history and simultaneous tumors in the colon, pelvis, and liver. These findings made diagnosis and treatment complicated. However, the multidisciplinary approaches followed by a medical oncologist, gynecologist, surgeon, radiologist, and pathologist led to a favorable outcome. This patient had two primary cancers of the colon and ovary, and systemic metastases of colon cancer. The loss of MSH6 protein expression was proven by immunohistochemical examination, but the germline MSH6 mutation was not detected by DNA
\end{abstract}

M. Shiono $\cdot$ H. Shimodaira $\cdot$ S. Akiyama $\cdot$ Y. Kakudo ·

S. Takahashi - M. Takahashi · C. Ishioka

Department of Clinical Oncology,

Tohoku University Hospital, Tohoku University,

1-1 Seiryo-machi, Aoba-ku, Sendai 980-8574, Japan

M. Watanabe

Department of Pathology, Tohoku University Hospital,

Tohoku University, 1-1 Seiryo-machi,

Aoba-ku, Sendai 980-8574, Japan

K. Takase

Department of Radiology, Tohoku University Graduate School

of Medicine, 1-1 Seiryo-machi, Aoba-ku,

Sendai 980-8574, Japan

K. Ito

Department of Disaster Obstetrics and Gynecology, International Research Institute of Disaster Science, Tohoku University,

1-1 Seiryo-machi, Aoba-ku, Sendai 980-8574, Japan sequence analysis. Regarding this discrepancy, some possibilities, e.g., genomic rearrangements and epigenetic modifications, which can be missed by conventional sequence analysis, were considered. Theoretically, Lynch syndrome cases with MSH6 impairment exhibit late onset and low penetrance compared to other major cases with $M L H 1$ or MSH6 mutations. Irinotecan hydrochloride (CPT-11) has favorable effects on MMR-deficient tumor cells with high microsatellite instability, although its clinical benefit remains controversial. In this case, the first-line chemotherapy bevacizumab + FOLFIRI regimen has been effective for over a year in the partial response state. We discuss the diagnostic, therapeutic, pathological, and molecular biological characteristics of this intriguing case, indicating the importance of family history, histological assessment, and molecular biological etiology in Lynch syndrome cases presenting a complicated phenotype.

\footnotetext{
K. Miura

Department of Surgery, Tohoku University Graduate School of Medicine, 1-1 Seiryo-machi, Aoba-ku, Sendai 980-8574, Japan

Y. Takami

Department of Hepato-Biliary-Pancreatic Surgery,

National Hospital Organization Kyushu Medical Center,

1-8-1 Jigyouhama, Chuo-ku, Fukuoka 810-8563, Japan

Y. Kakudo · C. Ishioka $(\bowtie)$

Department of Clinical Oncology, Institute of Development,

Aging, and Cancer, Tohoku University, 4-1 Seiryo-machi,

Aoba-ku, Sendai 980-8575, Japan

e-mail: chikashi@idac.tohoku.ac.jp

C. Ishioka

Cancer Center, Tohoku University Hospital, Tohoku University, 1-1 Seiryo-machi, Aoba-ku, Sendai 980-8574, Japan
} 
Keywords Lynch syndrome - Family history - MSH6 . Irinotecan (CPT-11) · Multiple cancers

\section{Case presentation}

Dr. Ishioka (medical oncologist, chairperson of the conference): Good evening, everyone. Today, we would like to discuss a thought-provoking Lynch syndrome case. (A brief summary of the case is given in the Abstract). Dr. Shiono, please begin the case presentation.

Dr. Shiono (medical oncologist, physician in charge of this case): A 51-year-old woman, diagnosed with advanced colon cancer with multiple liver metastases, was referred to our outpatient department by her primary practitioner for systemic chemotherapy.

The patient had been well until 3 weeks before a visit to her doctor for right upper quadrant pain. Abdominal ultrasound revealed multiple masses in the liver. Subsequent computed tomography (CT) revealed metastases from an unknown origin (Fig. 1a), and a pelvic mass was considered as a right ovarian mucinous cystadenoma (Fig. 1b). While esophagogastroduodenoscopy (EGD) detected no lesions, colonoscopy disclosed a type 2 tumor in the sigmoid colon, which was histologically diagnosed as a poorly differentiated adenocarcinoma (Fig. 1c). There was nothing in particular to declare in the patient's past medical and social histories. She had never been married or pregnant, and was post-menopausal. Her family history revealed a background of Lynch syndrome. Her father had been diagnosed with colorectal cancer at the age of 42 , and her two paternal uncles, aunt, and grandmother also had colorectal cancer (Fig. 2). This patient was diagnosed with Lynch syndrome by fulfilling the Amsterdam criteria II [1]. No apparent abnormalities were observed on physical examination. Laboratory data showed anemia $(\mathrm{Hb} 8.9 \mathrm{~g} / \mathrm{dl})$ and elevated CEA (1480 ng/ml) and CA19-9 (2418 U/ml) levels.

\section{Differential diagnosis}

To identify potential genes for Lynch syndrome, we submitted a colon cancer biopsy specimen for immunohistochemical (IHC) examination of the DNA mismatch repair (MMR) gene products, i.e., MLH1, MSH2, MSH6, and PMS2. Because Lynch syndrome was diagnosed, we could not completely rule out the possibility of ovarian cancer. An effective chemotherapy regimen should be selected based on the origin of the liver metastases. Hence, we consulted a radiologist and gynecologist for differential diagnoses of the pelvic tumor.
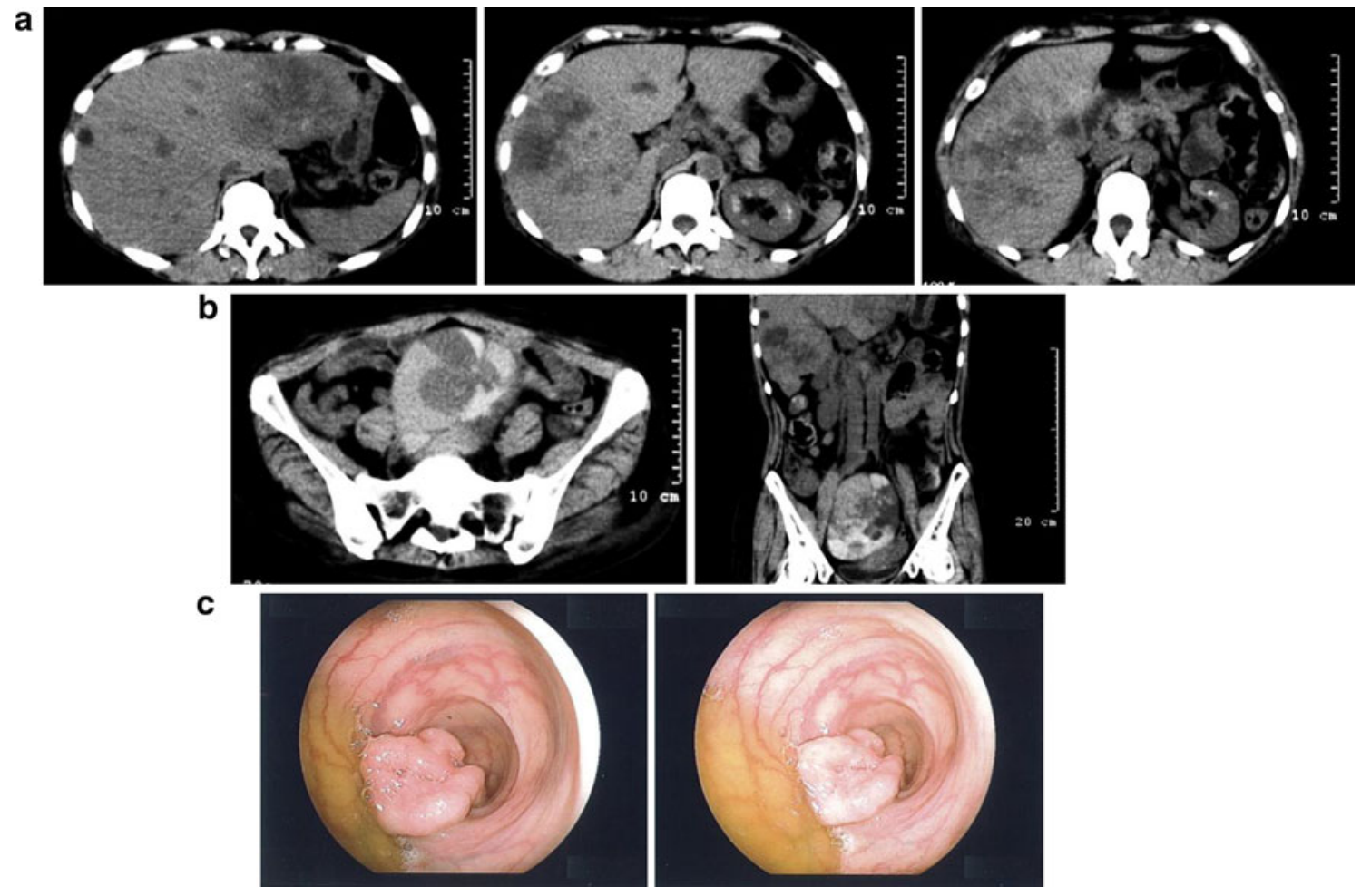

C

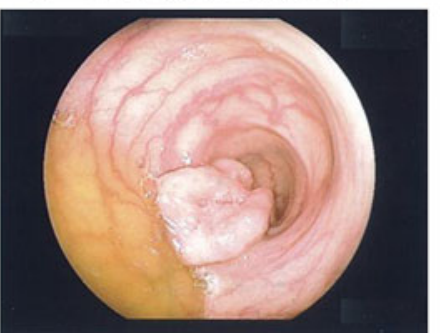

Fig. 1 CT images and colonoscopy findings at onset. a Axial images of the liver. Multiple low-density areas suggest metastases. b Axial and coronal images of the pelvic tumor. Various densities ranging from low to high, suggesting various liquid and solid components, are seen. c Captured images during colonoscopy. A massive type 2 tumor is seen in the sigmoid colon 
Fig. 2 Family tree. Five people were affected with colorectal cancer in the first degree relatives of the patient's father among three generations. Lynch syndrome was diagnosed, which fully met the diagnostic criteria of Amsterdam II. Squares and circles indicate male and female, respectively. Arrow indicates the patient. Filling with black indicates affected person with trait. Diagonal line indicates deceased relatives. $C R C$ colorectal cancer

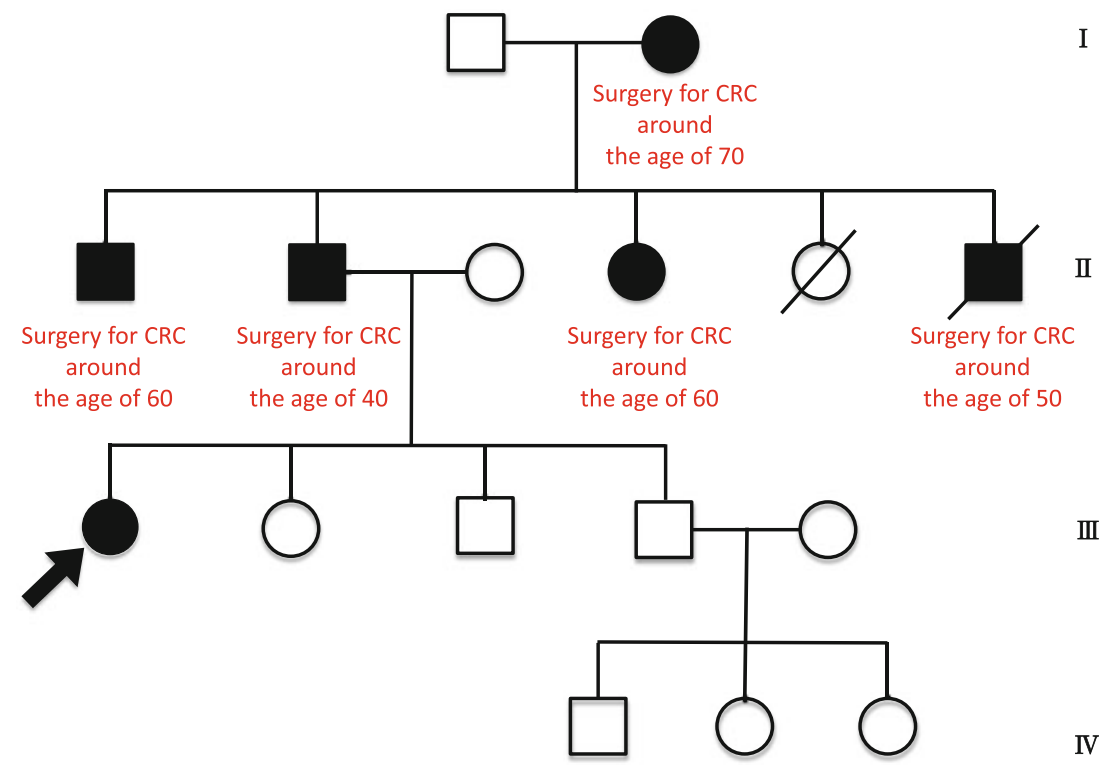

a
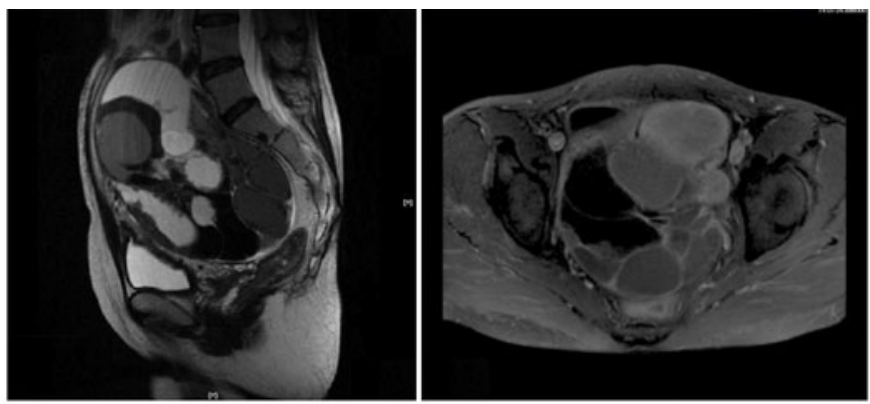

b
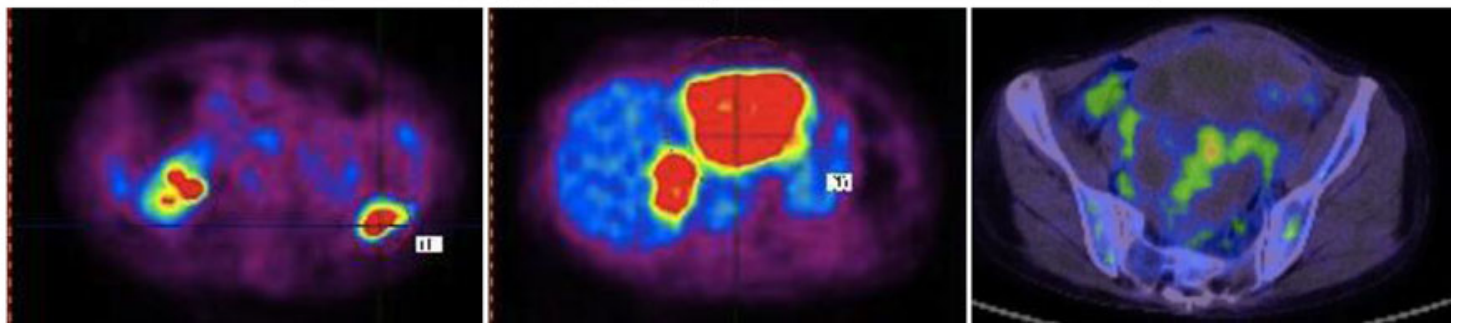

Fig. 3 PET-CT and MRI images 1 month after onset. a MRI images of the pelvic tumor. Mixtures of diverse intensities ranging from low to high, suggesting a variety of liquid and solid components, are seen. There seems to be a hemorrhage and mucus in it. Strong enhancement is seen in the cyst wall. A multilocular cystic ovary including a

Dr. Takase (radiologist): The pelvic mass was a multilocular cystic tumor, which showed various signals and densities on magnetic resonance imaging (MRI) and CT, respectively, presumably from a hemorrhage and mucus (Fig. 3a, left). Strong enhancement was observed in the cyst wall on MRI, although the solid part was minimal (Fig. 3a, right). A positron emission tomography/CT (PET-CT) image showed various maximum standardized uptake values showing malignancy $\left(\mathrm{SUV}_{\max } 9.0\right.$ in the colon, 7.0-10.0 in the liver, and 4.0 in the ovary) (Fig. 3b). It is difficult to determine whether an ovarian tumor is metastatic lesion of cyst walls with contrast enhancement was possibly suggested. b Axial PET-CT fusion images of the colon, liver, and ovary. $\mathrm{SUV}_{\max }$ values of 9.0 in the colon, 7-10.0 in the liver, and 4.0 in the ovary were detected

primary or metastatic when another definitive tumor is apparent [2, 3]. Most metastatic ovarian tumors show solid and cystic components, but a cyst is not evidence of primary ovarian cancer. Unlike metastatic tumors of other organs, it is common for metastatic ovarian tumors to contain cysts even if the primary site solely consists of a solid mass. When an ovarian cystic tumor and primary cancer are observed simultaneously, we first consider the possibility of a metastatic ovarian tumor. However, it was quite difficult to distinguish the masses through imaging. We thought that this might be a multilocular cystic ovary 
with metastatic lesions of the cyst walls with contrast enhancement [4].

Dr. Ito (gynecologist): Because few solid parts were present, which is often the case with primary ovarian cancer, in addition to normal CA-125 levels, a borderline tumor was conceivable in this case. However, laparotomy and histopathological assessment were necessary for the definitive diagnosis.

\section{Initial treatment plan and its course}

Dr. Shiono: Given the patient's history, we decided to prioritize chemotherapy for colorectal cancer, which had already been diagnosed as malignant. Considering her Lynch syndrome background, we selected an irinotecan hydrochloride (CPT-11)-based bevacizumab + FOLFIRI regimen. After confirming the uridine- 5 '-diphosphate-glucuronosyltransferase 1A1 (UGT1A1) *6 and *28 status as wild type for CPT-11 use, she was admitted for central venous port implantation for outpatient chemotherapy.

Because we used the biopsy specimens for MMR IHC, we performed colonoscopy to obtain biopsy samples for $K R A S$ gene mutation analysis.

Dr. S. Takahashi (medical oncologist, operator of colonoscopy): Compared to the photograph taken by the former endoscopist 2 months earlier, the tumor had grown so rapidly that the lumen was subtotally occluded (Fig. 4a). Taken together with the fact that colon-cleaning preparation required considerable time, stenosis seemed to be

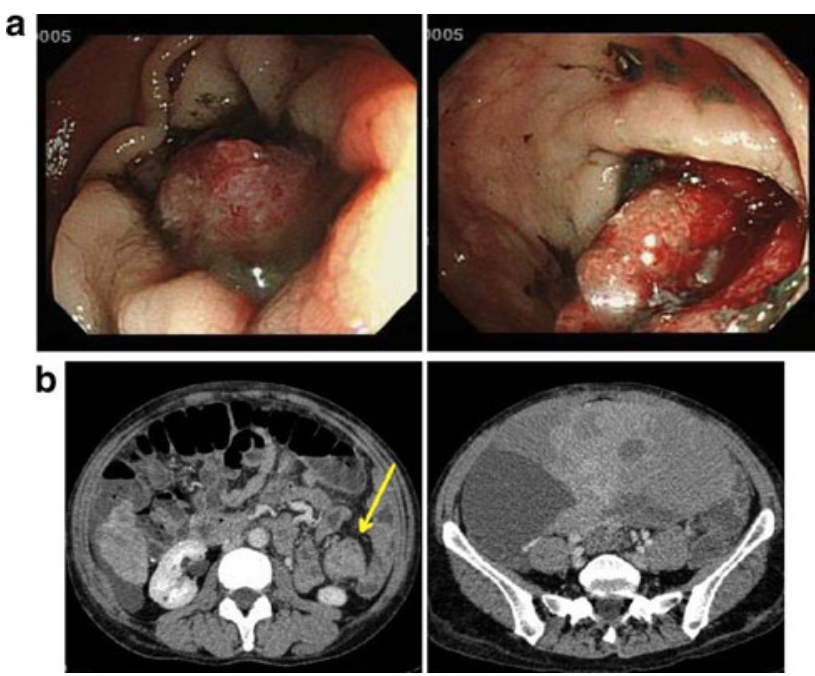

Fig. 4 Colonoscopy and CT images 2 months after onset. a Captured images during colonoscopy. Compared with Fig. 1c, the tumor had grown so rapidly that the lumen was subtotally occluded. b CT images of the colon and pelvic tumors. Left panel Upper colon from the stenosis site at the sigmoid with a massive tumor is enlarged. Arrow indicates the lesion. Right panel Pelvic tumor is also extremely increased in size compared with that in the former images severe. After acquiring the biopsy specimen, we performed $\mathrm{CT}$ to assess the indication for preemptive surgery for preventing mechanical colonic obstruction by the tumor.

Dr. Takase: The upper colon from the stenosis site at the sigmoid with massive tumor was enlarged. Compared with that in the CT images obtained at the former hospital, the ovarian tumor was also extremely enlarged (Fig. 4b).

Dr. Shiono: We consulted a surgeon for palliative surgery, planned elective operation, excluded bevacizumab to avoid interference with postoperative wound healing, and administered FOLFIRI chemotherapy ( $l$-LV 275 mg, CPT$11220 \mathrm{mg}$, 5-FU bolus i.v. $570 \mathrm{mg}$, 5-FU c.i.v. $3500 \mathrm{mg}$ ) once during the preoperative waiting period.

\section{Preoperative clinical diagnosis}

1. Lynch syndrome

2. Colorectal cancer

3. Ovarian tumor, borderline tumor suspected

4. Metastatic liver tumor

5. Subileus due to mechanical obstruction by colorectal cancer

Dr. Ishioka: Please tell us the operative findings, Dr. Miura.

Dr. Miura (surgeon): First, an infant head-sized multilocular and partially villous right ovarian tumor was seen. The left ovary had shrunk. In the abdominal cavity, the disseminated lesion and a small amount of pale yellow ascites were observed at vesicouterine and Douglas pouches, which were considered to be derived from right ovarian cancer. On the other hand, there was a near circumferential $50-\mathrm{mm}$ tumor in the middle portion of the descending colon. However, serous surface invasion was not recognized macroscopically. Multiple metastatic tumors were observed on the bilateral liver lobe, presenting the so-called state of "tumor liver." Because of diffuse intra-abdominal adhesions due to peritonitis carcinomatosa (PC) and definitive prognostic factors such as tumor liver or PC, we performed minimally invasive, palliative, and debulking surgery, i.e., descending colectomy, oophorectomy, and liver biopsy.

\section{Pathological discussion}

Dr. Ishioka: Dr. Watanabe, please explain the pathological findings.

Dr. Watanabe (pathologist): A circumferential type 2 tumor $(30 \times 25 \mathrm{~mm})$ was observed in the descending colon (Fig. 5a). This loupe image illustrates the part of the tumor penetrating the serosa (Fig. 5b). Histologically, a moderately differentiated tubular adenocarcinoma (tub2) 

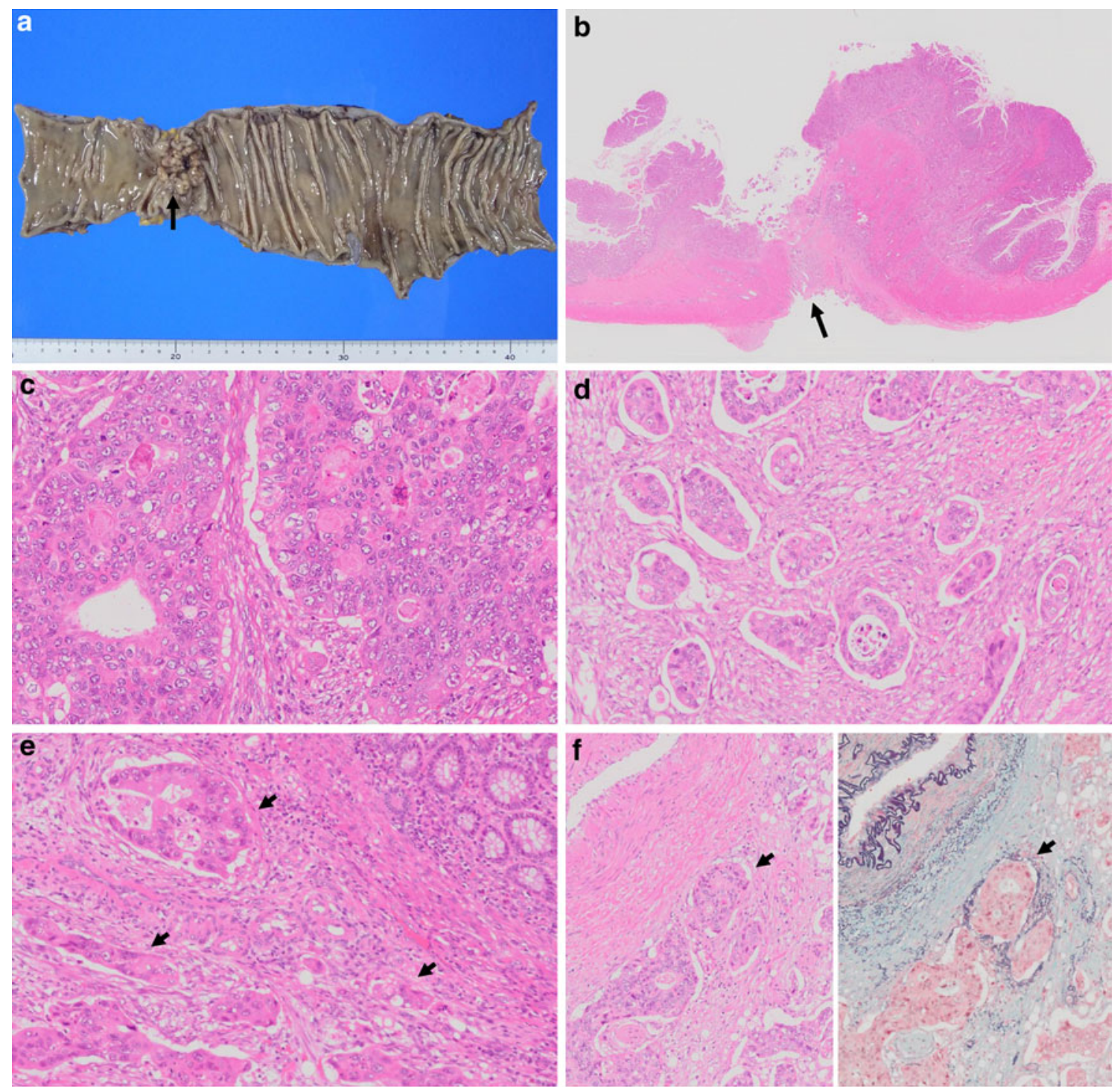
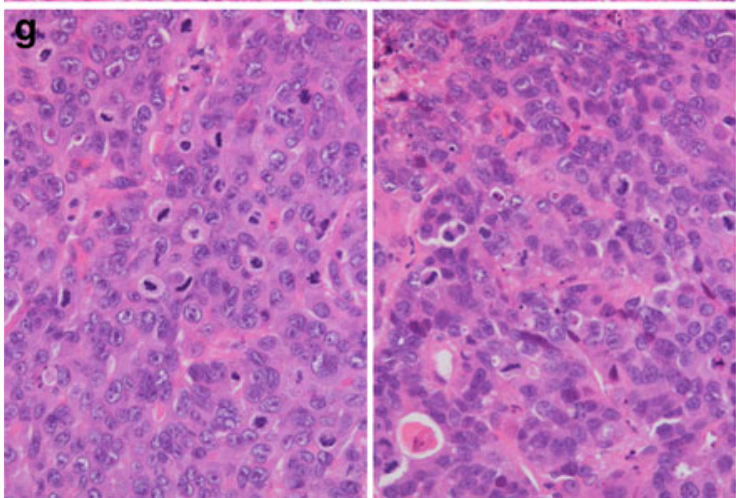

Fig. 5 Pathological findings of the descending colon cancer. a Macroscopic view of the resected descending colon with the cancer. A circumferential type 2 tumor $(30 \times 25 \mathrm{~mm})$ is seen. b Loupe view. Arrow indicates the part where cancer cells penetrated the serosa. c Detailed microscopic view. Moderately differentiated tubular adenocarcinoma (tub2) with a cribriform pattern is apparent. There is not much difference compared with conventional colorectal cancer. d An invasive micropapillary carcinoma pattern (IMPC). Nesting

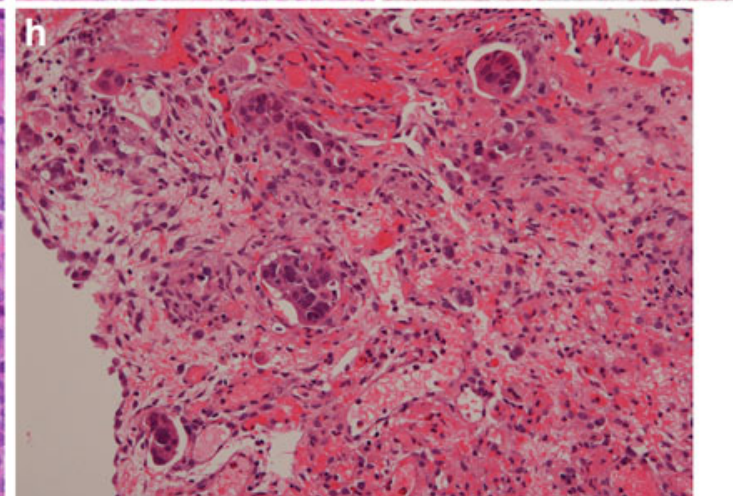

cancer cells within spaces separating themselves from the surrounding stroma is seen in the invasive area. e Lymphatic involvement. Arrows indicate the parts. f Venous involvement. Left panel H\&E stain. Arrow indicates the parts. Right panel Elastica-Masson stain for veins. Arrow indicates the same parts of the left panel. Liver (g) and peritoneal (h) metastases. The histologically similar, moderately differentiated, tubular adenocarcinomas originated from primary colon cancer are seen 
with a cribriform pattern was apparent, which was not much different compared with conventional colorectal cancer (Fig. 5c). An invasive micropapillary carcinoma pattern (IMPC), which has nesting cancer cells within spaces separating them from the surrounding stroma, was seen in the invasive area (Fig. 5d). You can recognize the lymphatic involvement (1y3, Fig. 5e) and venous permeation (v2, Fig. 5f). Metastases of the same moderately differentiated tubular adenocarcinoma were seen in the liver and peritoneum (Fig. 5g, h). Thus, the pathological diagnosis for colon cancer was "Japanese Classification of Colorectal Carcinoma: D, type 2, $30 \mathrm{~mm}$, tub2, pSE, int, $\mathrm{INFb}, \mathrm{ly} 3$, v2, bud(-), pPM0, pDM0, pN1 (1/15), pH1 (grade A), pP1, Cy1, cM0, stage IV; TNM classification: pT4a, pN1a, pM1b, G2, stage IVB.”

Although it was difficult to decide between primary or metastatic based on resemblance, we finally diagnosed primary ovarian cancer. The right ovary $(180 \times 150 \mathrm{~mm})$ consisted of cystic and solid parts (Fig. 6a). A loupe view showed papillary or solid tumor growths beside wide necrotic lesions in the cyst (Fig. 6b). Columnar atypical cells with tubular formation similar to that of colon cancer were also observed (Fig. 6c). An ovarian metastatic tumor can morphologically resemble primary ovarian cancer [5], and the colon is regarded as a primary lesion [6]. Therefore, it is feasible to presume that the ovarian tumor was metastasis of the colon cancer. However, there was evidence of primary ovarian cancer. You can recognize a definitive transitional lesion from benign epithelium to an atypical one (Fig. 6d). This "in situ lesion" is clearly primary.

In IHC studies, both colon and ovarian tumor cells showed CA125(-), CA19-9(+), vimentin(-), CK7(-), CK20(+), CDX2(+), resembling colorectal cancer staining patterns (Fig. 6e, f). Yet, the characteristic difference between them was the staining pattern of PTEN, which supports the likelihood of ovarian serous adenocarcinoma (Table 1). Hence, the ovarian tumor was diagnosed as "serous adenocarcinoma, TNM classification: pT1c, cN0, cM0, G1, FIGO stage IC."

The features of Lynch syndrome-related ovarian cancer are as follows: young onset (mean age 48 years), early stage (FIGO stage I, $47 \%$ ), comparatively frequent seroustype histology (endometrioid $35 \%$, serous $28 \%$, clear cell $17 \%$, mucinous $5 \%$, undifferentiated $15 \%$ ), and high attribution rate of MSH6 deficiency among underlying MMR gene mutations (MSH2 $49 \%$, MSH6 $33 \%$, MLH1 $17 \%$ ) [7].

Dr. Ishioka: Please describe the MMR IHC results.

Dr. Shimodaira (medical oncologist): Whereas MLH1, MSH2, and PMS2 showed nuclear staining patterns indicating intact expression, MSH6 did not (Fig. 7). Thus, MSH6 must be responsible gene for this case.
Fig. 6 Pathological findings of the right ovarian cancer. a Macroscopic view of the resected right ovary with the cancer. It is $180 \times 150 \mathrm{~mm}$ in size and consisted of cystic and solid parts. b Loupe view. Papillary or solid growths of the tumor besides wide necrotic lesions are seen in the cyst. c Detailed microscopic view. Some columnar atypical cells with tubular formation similar to that of colon cancer were seen. d In situ lesion. There are epithelial cells overlaying the inner surface of the cyst, which shows definitive transitional lesions from benign epithelium to an atypical one. The in situ lesion is evidence of primary ovarian cancer. e IHC studies for CK7 (left upper and right upper panels) and CK20 (left lower and right lower panels). Left upper and left lower panels Colon cancer. Right upper and right lower panels Ovarian cancer. f IHC studies for CDX2 (left upper and right upper panels) and CA125 (left lower and right lower panels). Left upper and left lower panels Colon cancer. Right upper and right lower panels Ovarian cancer

Dr. Ishioka: What were the results of sequence analysis?

Dr. Shiono: Despite the above-mentioned IHC results of the colon cancer specimen, we could not detect any pathogenic germline mutations in $M L H 1, M S H 2$, and $M S H 6$ by direct sequence analyses. The mechanism of that divergence was unclear and will be discussed later.

Moreover, the genetic status of KRAS was wild type with regard to inspected codons 12 and 13 .

Dr. Miura: That was very interesting. Concerning the differential diagnosis of the pelvic tumor, many organs could be the candidate origin, e.g., colon, bladder, prostate, ovary, and uterus. Lastly, we proposed IHC marker sets as the screening criteria [8]. Although these sets seemed unnecessary in this case because detailed molecular analyses had already been performed, they might be useful in other cases depending on the situation.

\section{Final diagnosis}

1. Lynch syndrome with MSH6 deficiency

2. Descending colon cancer (tub2, pT4a, pN1a, pM1b, stage IVB) with multiple metastases to the lymph nodes, liver, and peritoneum

3. Right ovarian cancer (serous adenocarcinoma, pT1c, cN0, cM0, G1, FIGO stage IC)

\section{Clinical course}

Dr. Ishioka: Well, tell us the clinical course after that, please.

Dr. Shiono: The postoperative course was favorable. The first visit day after discharge to restart bevacizumab + FOLFIRI therapy was 11 March 2011. While in the waiting room of the Tohoku University Hospital Cancer Center, the Great East Japan Earthquake occurred. Because she resided in the coastal area, she lost her house in the tsunami. Therefore, she moved to Fukuoka 

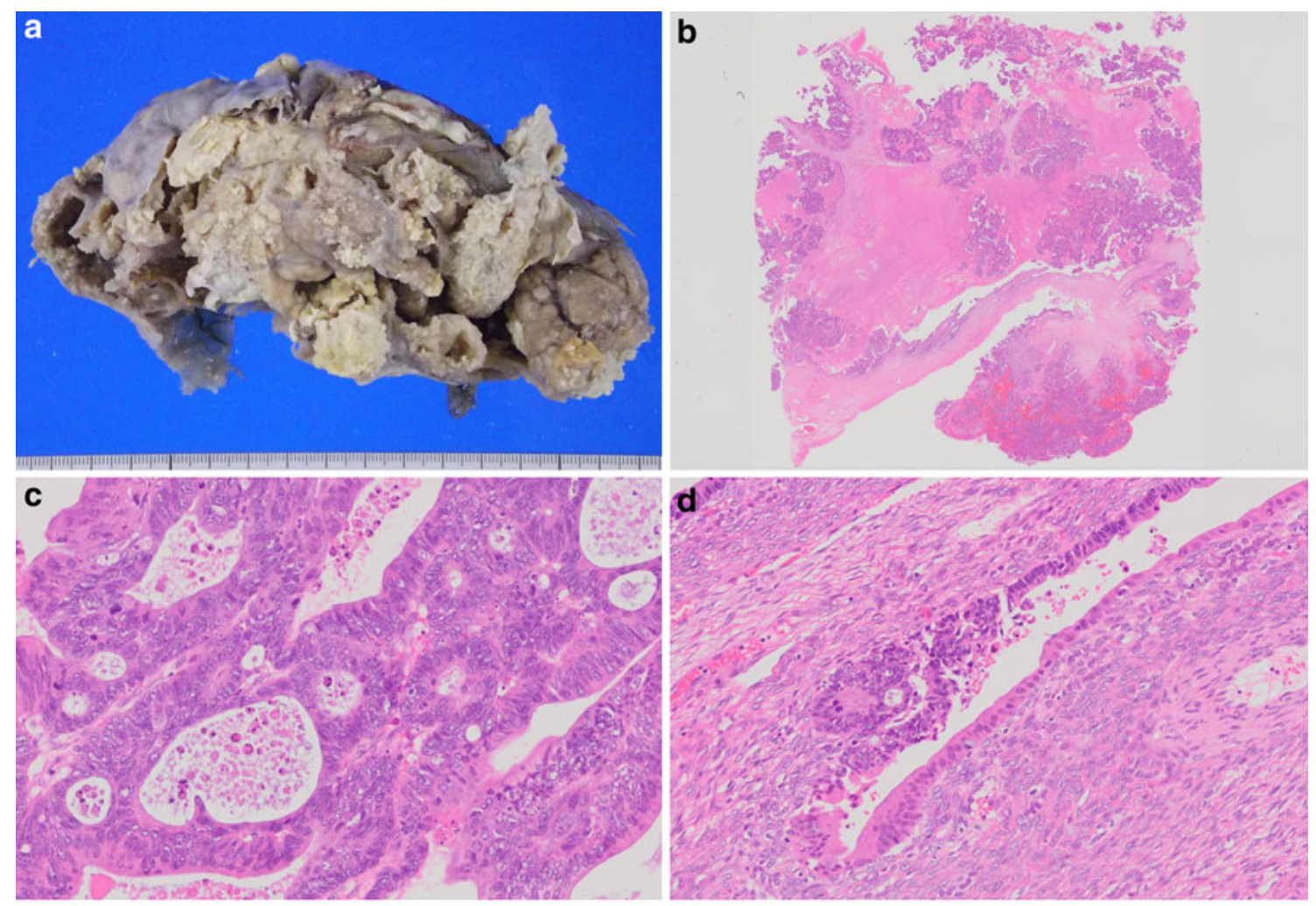

Site :

Colon

Ovary
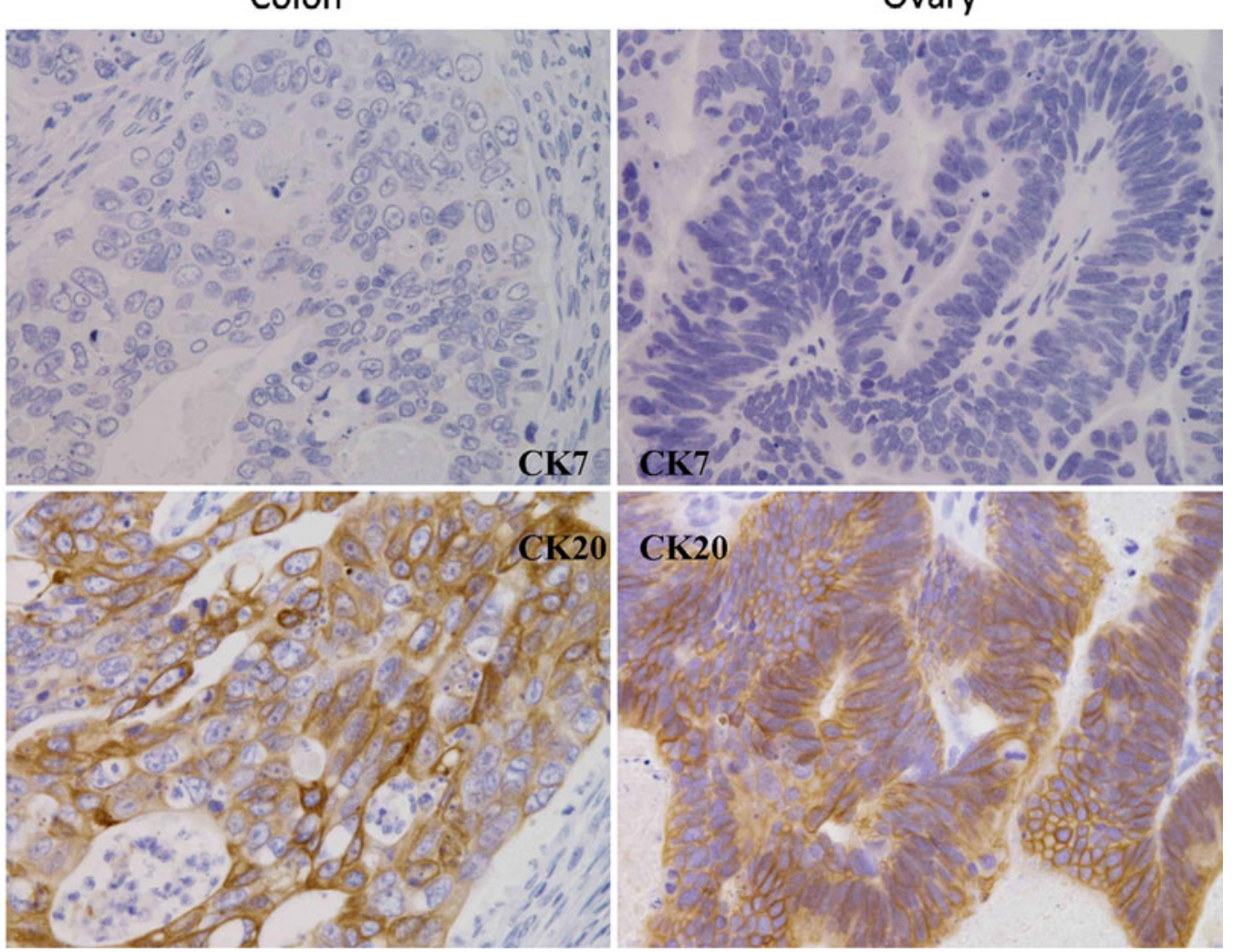


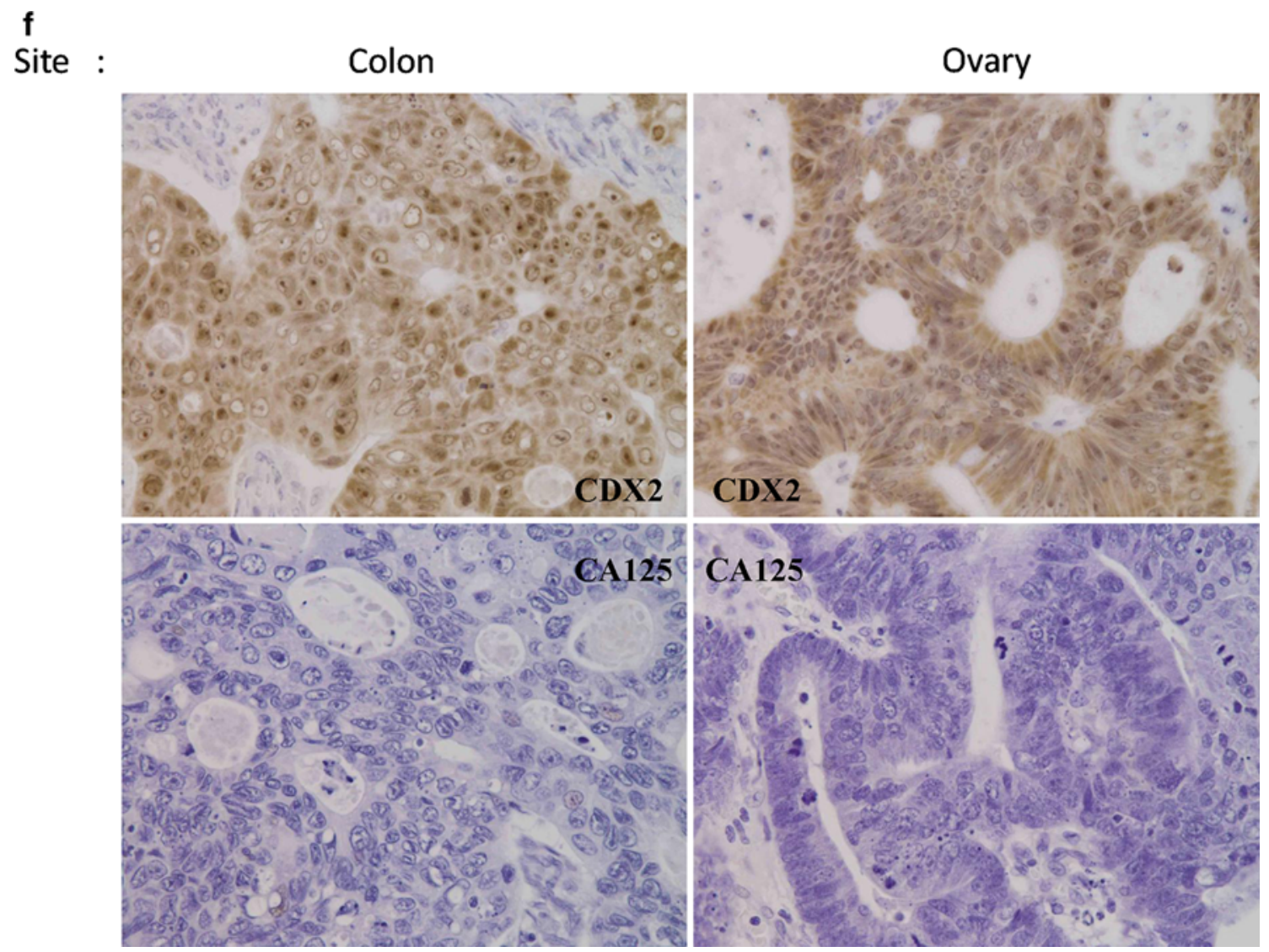

Fig. 6 continued

Table 1 Immunohistochemistry of colon and ovarian carcinomas

\begin{tabular}{lll}
\hline & Colon & Ovary \\
\hline CK7 & - & - \\
CK20 & + & + \\
CDX2 & + & + \\
CA125 & - & - \\
CEA & ++ & ++ \\
ER & - & - \\
PgR & - & - \\
p53 & + & + \\
p16 & Focal+ & Focal+ \\
PTEN & Focal+ & Diffuse++ \\
Vimentin & - & - \\
\hline
\end{tabular}

prefecture on Kyushu Island with relatives. Fortunately, she restarted the same chemotherapy at the National Hospital Organization Kyushu Medical Center (Bev $230 \mathrm{mg}, \quad l$-LV $275 \mathrm{mg}$, CPT-11 $200 \mathrm{mg}$, 5-FU bolus $560 \mathrm{mg}$, 5-FU civ. $3000 \mathrm{mg}$ ). She has remained in the partial response (PR) state over a year.

Dr. Ishioka: We have a comment from Dr. Takami, who is in charge at Kyushu Medical Center. Please read it for us.
Dr. Shiono (reading Dr. Takami's comment): The patient suddenly came to our hospital without any medical information on 22 March. Luckily, a phone line to Tohoku University Hospital was available on that day after the disaster, and I spoke to Dr. Shiono. After receiving a detailed referral form, we immediately initiated bevacizumab + FOLFIRI administration based on the diagnosis and proposed dose from 29 March. Fortunately, the chemotherapy has been effective. The tumor marker levels and tumor sizes of the liver metastases have decreased dramatically (Fig. 8a, b). One year later, she is still receiving benefits from first-line chemotherapy, which is amazing considering her status of severe metastases.

\section{Discussion}

Dr. Ishioka: Let's move on to the discussion.

Dr. Shiono: First, it was challenging to determine whether the liver metastases originated from the colon or ovary because of the difficulty in the differential diagnosis of the pelvic tumor. In this case, the clinical response to the regimen was favorable, which was consequently in line with the histopathological assessment obtained via 


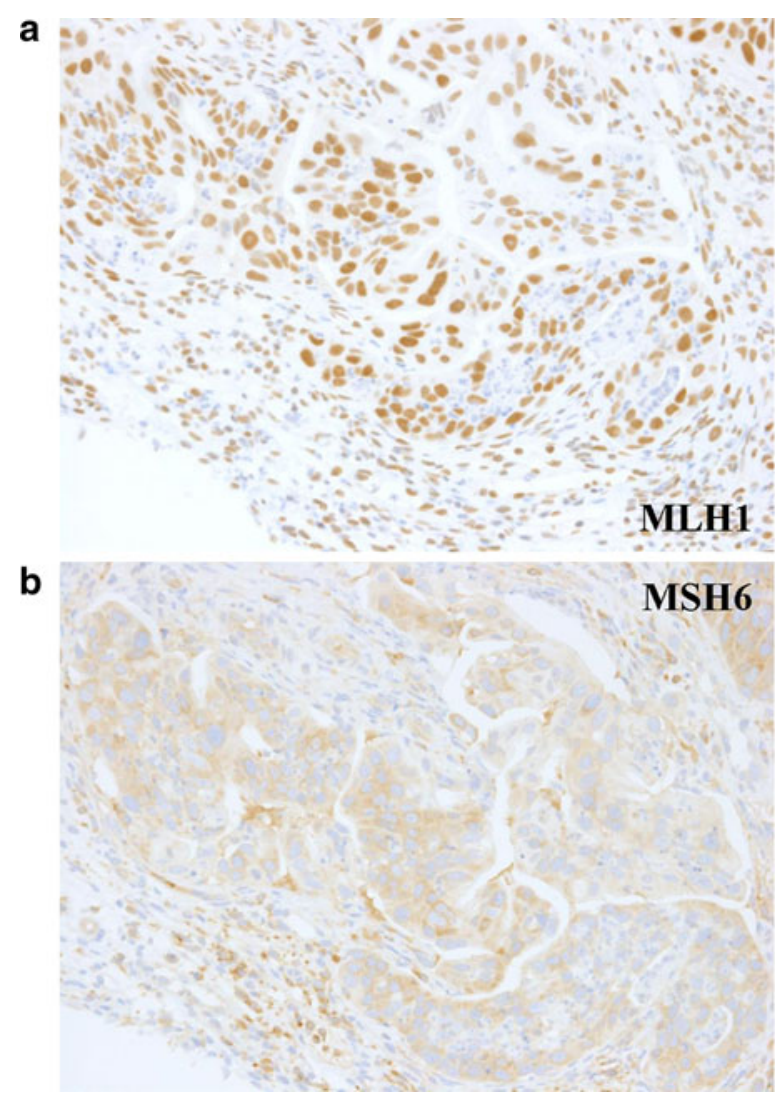

Fig. 7 IHC for MMR proteins using the colon cancer specimen. a, b IHC studies for MLH1 (left upper panels), MSH2 (right upper panels), MSH6 (left lower panels), and PMS2 (right lower panels). Whereas MLH1, MSH2, and PMS2 showed the nuclear staining

palliative surgery. Thus, it is important to select a suitable regimen in accordance with the histology, if possible.

Dr. Ishioka: What about the practical treatment?

Dr. Shiono: In the literature, CPT-11 is clearly effective on MMR-deficient tumor cells in vitro and favorable in some clinical studies, but its clinical evidence remains controversial [9-17]. Hence, we selected the bevacizumab + FOLFIRI regimen as first-line chemotherapy for the metastatic colorectal cancer. In retrospect, because it has still been effective in the PR state for over a year, the chemotherapy choice seems to be reasonable in this case.

Dr. Ishioka: Please explain the standard first-line chemotherapy for advanced or metastatic colorectal cancer, Dr. Kakudo.

Dr. Kakudo (medical oncologist): There are some options. As first line chemotherapy, we choose FOLFIRI or FOLFOX (5-FU/l-LV/l-OHP) regimens as a combination of cytotoxic agents. Sequential therapy like FOLFIRI or FOLFOX regimen as first line, followed by the alternative regimen as second line, has improved outcome regardless of the order of the regimens [18]. CapeOX, the regimen using the oral prodrug of 5-FU, is another option showing

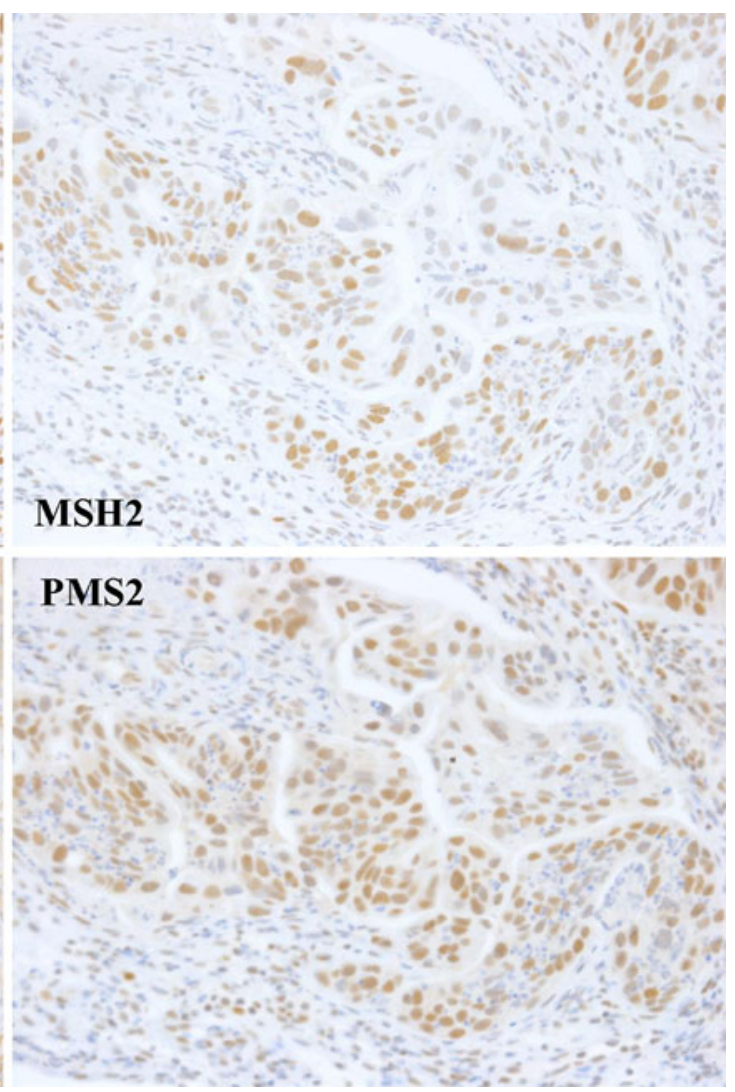

pattern, MSH6 did not. Left lower panel Cytoplasmic weak and blur staining was regarded as non-specific compared to other positive and negative controls

an almost identical outcome compared to FOLFOX [19]. The common adverse events are different: peripheral neuropathy in l-OHP and diarrhea in CPT-11. The last choice is whether to add molecular-targeted agents such as bevacizumab (anti-VEGF antibody drug) or cetuximab/panitumumab (anti-EGFR antibody drugs). You should pay attention to contraindications of these monoclonal antibody drugs, e.g., the comorbid severe vascular problems in bevacizumab use. Patients with the KRAS gene mutation should be excluded from cetuximab/panitumumab administration. We make an optimal decision depending on the circumstances of each case [20,21].

Dr. Shiono: In this case, CPT-11 was used as a key drug considering the Lynch syndrome background. CPT-11's effectiveness against cancer cells resulting from a MMR deficiency has been demonstrated in in vitro analyses. Although the entire mechanism remains unclear, it is speculated that CPT-11, an topoisomerase-I inhibitor, exerts its cytotoxicity by generating DNA double-strand breaks (DSBs) in the administered cell. Conversely, MMRdeficient tumor cells have a tendency to accumulate mutations within microsatellite repeats of genes associated 


\section{a}

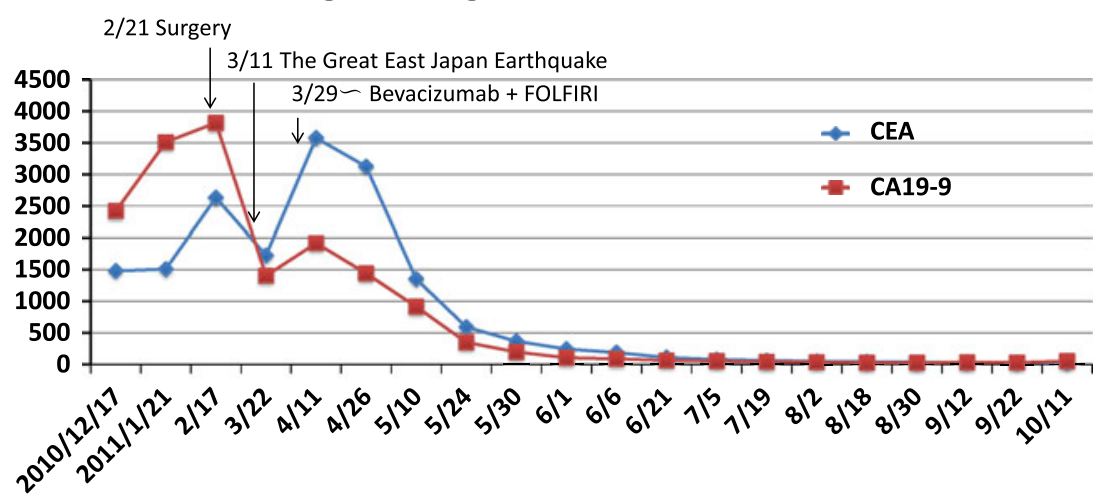

b

Date : $\quad 2 / 7$

$4 / 5$

$6 / 21$

$9 / 9$

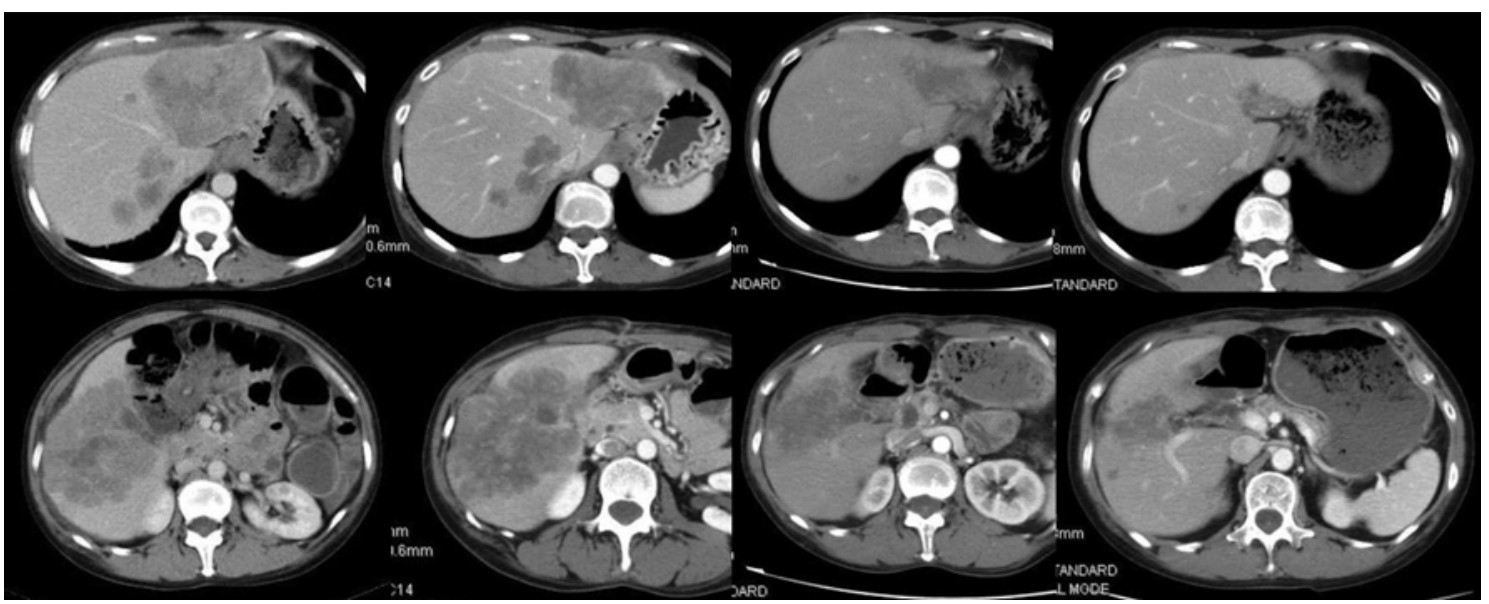

Fig. 8 Therapeutic effect of the systemic bevacizumab + FOLFIRI therapy. a Chronological change in the tumor marker levels. They decreased dramatically after administration of the systemic bevacizumab + FOLFIRI therapy. b Chronological change in liver metastases. They have decreased dramatically after administration of systemic bevacizumab + FOLFIRI therapy and have been in the PR state over a year with DSBs repair, such as MRE11 and RAD50. Taken together, MMR-deficient cells exhibit high sensitivity to CPT-11 [9-17]. Hence, CPT-11 treatment might be effective in patients with microsatellite instability-high (MSI-H) colorectal cancer.

Dr. M. Takahashi (medical oncologist): In fact, a previous randomized study for adjuvant chemotherapy against stage III MSI-H colon cancers demonstrated the significant advantage of the addition of CPT-11 [12]. However, another subsequent study did not reveal the benefit in a similar population [13]. Thus, the clinical benefit of CPT11 compared with other agents in MSI-H colorectal cancer remains controversial. Because the mutations in MRE11 or $R A D 50$ are detected in many but not all MSI-H tumors (70-85\%) [22], the MSI-H phenotype may not always correlate with the hypersensitivity of tumors to CPT-11. Another marker to indicate MRE11 or RAD50 deficiency (e.g., mutational analysis and/or IHC) may help to predict the efficacy of the CPT-11 treatment.

Dr. Ishioka: As both the former trials (CALGB89803 and PETACC-3) were adjuvant trials for stage II/III colorectal cancer $[12,13,23]$, the clinical relevance of the outcome was slightly different in this stage IV case. While the evidence level of the advantage of CPT-11 for MSI-H tumor was not sufficient, selecting FOLFIRI among the standard therapies was reasonable according to the concept that the most promising therapy should be given priority. Moreover, when you use CPT-11, you must evaluate the patient for the UGT1Al gene polymorphism. Dr. Akiyama, please offer a general explanation.

Dr. Akiyama (medical oncologist): CPT-11 is inactivated by UGT1A1. If a specific gene polymorphism exists in UGT1A1, the glucuronidation level of SN-38, the active metabolite of CPT-11, would be attenuated, resulting in 
drug accumulation and toxicity enhancement; this leads to diarrhea, neutropenia, etc. To be more precise, homozygosity for $U G T I A I * 28$ or $U G T 1 A 1 * 6$ and heterozygosity for both $U G T I A I * 6$ and $U G T I A I * 28$ are the polymorphisms mentioned in the package insert of the drug. However, optimal criteria for dosage adjustments have not been established. Moreover, there are some differences among ethnicities. In Asians, $U G T I A I^{*} 6$ is more frequent than $U G T 1 A 1 * 28$. Conversely, UGTIAI*28 is much more common than $U G T 1 A I^{*} 6$, which is quite rare in Caucasians and African-Americans. Such discordance is derived from the different genetic background among the races [24-26].

Dr. Ishioka: With regard to dose, $150 \mathrm{mg} / \mathrm{m}^{2}$ is defined as the maximal dose in Japan, although $180 \mathrm{mg} / \mathrm{m}^{2}$ is the standard in Europe and the US. Accordingly, data from overseas cannot be used for direct comparisons. Many research groups, including ours, are working on this topic, and an appropriate criterion for the Japanese people needs to be established. Well, let us get back to this case. What about MSI in this case? Would you explain the reason, if you did not check?

Dr. Shiono: We obtained positive IHC results, and therefore, we did not perform an MSI examination. IHC is the best initial examination because it directs the candidate gene for subsequent mutation analysis in families with a high probability of having a mutation (the revised Bethesda guidelines or Amsterdam II criteria) [27]. Moreover, the latest analysis on the accuracy and cost-effectiveness of IHC and/or MSI examination to screen for Lynch syndrome [28] promotes the following strategies: "IHC and MSI performed simultaneously" and "IHC followed by MSI if IHCs were normal." The latter was slightly better in terms of cost. Therefore, IHC seems to be sufficient if it is performed first. According to this strategy, if IHC demonstrated the candidate mutated gene, you can skip MSI and proceed to direct sequencing. IHC has an advantage in terms of specifying the putative mutated MMR gene compared with MSI [29].

Dr. Ishioka: OK, so it is reasonable. However, how do you explain the discrepancy between the results of IHC and sequence analyses?

Dr. Shiono: As seen in Fig. 7b, nuclear staining of MSH6 alone was lost compared with that of the other three MMRs. Some possibilities were considered. For example, it is known that genomic rearrangements such as large deletions cannot be detected by conventional sequence analysis [30-32], actually in a significant proportion of Lynch syndrome families (5-20 \%) [33-36]. Otherwise, it may be a type of epigenetic modification such as methylation [37]. However, further molecular analyses, e.g., the multiplex ligation-dependent probe amplification (MLPA) test, are needed for elucidation [31, 33, 38].
Dr. Ishioka: What about care for the families because this is a hereditary syndrome?

Dr. Shiono: Complying with the guidelines [39, 40], we performed a genetic counseling series for the patient, and her sister wished to accompany her. Her siblings shared the information, recognized the importance of medical follow-up, and have begun to undergo annual screening examinations, including colonoscopy. You can refer to the surveillance recommended by the international collaborative groups [27, 41]. However, a study indicated that the screening recommendations for MSH6 mutation carriers may slightly differ from those for Lynch syndrome carriers as a whole, reflecting the characteristics of MSH6-mutated Lynch syndrome $[42,43]$. The weaker phenotype, which is observed as a result of MSH6 mutations, exhibits a later age of onset and lower penetrance compared with that observed as a result of $M L H 1$ or $M S H 2$ mutations [44, 45]. Many types of cancer should be considered in regard to an increased risk, e.g., cancer of the colorectum, endometrium, stomach, small intestine, hepatobiliary system, kidney, urinary bladder, brain, and ovary. The latest prospective study showed that pancreatic and breast cancers had an elevated risk [46].

Dr. Ishioka: Finally, what is the discriminative point in this case compared with other Lynch syndrome cases?

Dr. Shiono: In general, approximately $90 \%$ of Lynch syndrome cases with mutations in any MMR genes are attributed to $M L H 1$ or $M S H 2$ mutations with distinct clinical features such as early onset $(<50$ years) and proximal colon predominance [47-52]. In contrast to these characteristics, it might have been difficult to diagnose Lynch syndrome in this case without a definitive family history. Moreover, with respect to comorbid cancer, while the frequency of endometrial cancer is as high as $60-70 \%$, the frequency of ovarian cancer is only 7-10\% [53]. Hence, clinical information on ovarian lesions might be relatively less likely to indicate Lynch syndrome. As mentioned above, although the incidence of Lynch syndrome attributed to MSH6 mutation is as low as approximately $10 \%$, it is known to show "relatively late onset" and "low penetrance" propensity compared with $M L H 1$ or $M S H 2$ mutations [42, 44, 45]. Thus, judging by only clinical manifestation may lead to a diagnostic pitfall. To avoid misdiagnosis of Lynch syndrome, considering a family history is always critically important.

Dr. Shimodaira: Concerning the unique phenotype of MSH6-deficient Lynch syndrome, the mechanism can be understood when the molecular function of the four MMR proteins is considered. First, they function as heterodimers formed by MSH2 in association with MSH6 (MutS $\alpha$ ) or MSH3 (MutS $\beta$ ) and MLH1 interaction with PMS2 (MutL $\alpha$ ), respectively. As seen in these complexes, the contribution of MSH6 is relatively small compared with that of major players such as MLH1 or MSH2, which 
interact with many gene products. In fact, MSH6 functionally participates only in detection of single-base mismatch or small loop-out mutations, while MLH1 or MSH2 engages in widespread mismatches other than single-base abnormalities. Thus, the loss of MSH6 function involves only a partial deficiency of the MMR system, and subsequently it results in an attenuated clinical phenotype, which is approximated to conventional colorectal cancers with regard to late onset and low penetrance, compared with those caused by MLH1 or MSH2 deficiency [54, 55].

Dr. Shiono: In conclusion, this was a very intriguing discussion on diagnostics, treatment, pathology, and molecular biology. It is also dramatic that she was saved from the tsunami, which deprived her of her house on the seashore, by an occasional visit to our hospital during the Great East Japan Earthquake. She has also been spared from life-threatening disease progression by treatment based on the cooperation of many doctors. I appreciate all your kind collaborative work.

Dr. Ishioka: The first-line bevacizumab + FOLFIRI treatment exerted a pronounced effect on this metastatic case of MSH6-mutated Lynch syndrome. The population of Lynch syndrome cases with metastasis is too small to organize a large-scale randomized prospective trial in order to elucidate CPT-11 effectiveness. However, the prevalence of MSI among all colorectal cancers is approximately $15 \%$ $[56,57]$. Therefore, it might be possible to conduct a clinical trial by alternatively targeting similar types of cancers. Thus, further analysis is needed to elucidate the clinical benefit of the drug. Are there any questions? Then, this conference is adjourned. Thank you for your attendance.

\section{What we learned from this case conference}

1. You must always collect detailed information regarding family history in order not to overlook familial tumor syndromes.

2. You should know that weaker phenotypes, such as "late onset" and "low penetrance," compared to MLH1- or MSH2-mutated Lynch syndrome, can be observed because of MSH6 deficiency.

3. A histopathological diagnosis must be obtained as soon as possible before deciding on an optimal regimen for patients with multiple primary cancers.

4. Although it is controversial at the clinical level and requires further study, a CPT-11-based regimen may have favorable effects on Lynch syndrome cases, depending on MMR deficiency.

Conflict of interest Honoraria: C. Ishioka, Taiho Pharmaceutical Co. Ltd.; C. Ishioka received research funding form Chugai Pharmaceutical Co., Ltd.

\section{References}

1. Vasen HF, Watson P, Mecklin JP et al (1999) New clinical criteria for hereditary nonpolyposis colorectal cancer (HNPCC, Lynch syndrome) proposed by the International Collaborative group on HNPCC. Gastroenterology 116:1453-1456

2. Brown DL, Zou KH, Tempany CM et al (2001) Primary versus secondary ovarian malignancy: imaging findings of adnexal masses in the Radiology Diagnostic Oncology Group Study. Radiology 219:213-218

3. Imaoka I, Wada A, Kaji Y et al (2006) Developing an MR imaging strategy for diagnosis of ovarian masses. Radiographics 26:1431-1448

4. Kim SH, Kim WH, Park KJ et al (1996) CT and MR findings of Krukenberg tumors: comparison with primary ovarian tumors. J Comput Assist Tomogr 20:393-398

5. Garcia A, De la Torre J, Castellvi J et al (2004) Ovarian metastases caused by cholangiocarcinoma: a rare Krukenberg's tumour simulating a primary neoplasm of the ovary: a two-case study. Arch Gynecol Obstet 270:281-284

6. Lash RH, Hart WR (1987) Intestinal adenocarcinomas metastatic to the ovaries. A clinicopathologic evaluation of 22 cases. Am J Surg Pathol 11:114-121

7. Ketabi Z, Bartuma K, Bernstein I et al (2011) Ovarian cancer linked to Lynch syndrome typically presents as early-onset, nonserous epithelial tumors. Gynecol Oncol 121:462-465

8. Miura K, Ishida K, Fujibuchi W et al (2012) Differentiating rectal carcinoma by an immunohistological analysis of carcinomas of pelvic organs based on the NCBI Literature Survey and the Human Protein Atlas database. Surg Today 42(6):515-525

9. Vilar E, Scaltriti M, Balmana J et al (2008) Microsatellite instability due to hMLH1 deficiency is associated with increased cytotoxicity to irinotecan in human colorectal cancer cell lines. Br J Cancer 99:1607-1612

10. Fallik D, Borrini F, Boige V et al (2003) Microsatellite instability is a predictive factor of the tumor response to irinotecan in patients with advanced colorectal cancer. Cancer Res 63:57385744

11. Jacob S, Aguado M, Fallik D et al (2001) The role of the DNA mismatch repair system in the cytotoxicity of the topoisomerase inhibitors camptothecin and etoposide to human colorectal cancer cells. Cancer Res 61:6555-6562

12. Bertagnolli MM, Niedzwiecki D, Compton CC et al (2009) Microsatellite instability predicts improved response to adjuvant therapy with irinotecan, fluorouracil, and leucovorin in stage III colon cancer: Cancer and Leukemia Group B Protocol 89803. J Clin Oncol 27:1814-1821

13. Tejpar S, Bosman F, Delorenzi M, Fiocca R, Yan P, Klingbiel D et al (2009) Microsatellite instability (MSI) in stage II and III colon cancer treated with 5FU-LV or 5FU-LV and irinotecan (PETACC 3-EORTC 40993-SAKK 60/00 trial). J Clin Oncol 27:(suppl; abstr 4001)

14. Bras-Goncalves RA, Rosty C, Laurent-Puig P et al (2000) Sensitivity to CPT-11 of xenografted human colorectal cancers as a function of microsatellite instability and p53 status. Br J Cancer 82:913-923

15. Giannini G, Rinaldi C, Ristori E et al (2004) Mutations of an intronic repeat induce impaired MRE11 expression in primary human cancer with microsatellite instability. Oncogene 23:26402647

16. Magrini R, Bhonde MR, Hanski ML et al (2002) Cellular effects of CPT-11 on colon carcinoma cells: dependence on p53 and hMLH1 status. Int J Cancer 101:23-31

17. Miquel C, Jacob S, Grandjouan S et al (2007) Frequent alteration of DNA damage signalling and repair pathways in human 
colorectal cancers with microsatellite instability. Oncogene 26: 5919-5926

18. Tournigand C, Andre T, Achille E et al (2004) FOLFIRI followed by FOLFOX6 or the reverse sequence in advanced colorectal cancer: a randomized GERCOR study. J Clin Oncol 22:229-237

19. Cassidy J, Clarke S, Diaz-Rubio E et al (2008) Randomized phase III study of capecitabine plus oxaliplatin compared with fluorouracil/folinic acid plus oxaliplatin as first-line therapy for metastatic colorectal cancer. J Clin Oncol 26:2006-2012

20. Chibaudel B, Tournigand C, Andre T et al (2012) Therapeutic strategy in unresectable metastatic colorectal cancer. Ther Adv Med Oncol 4:75-89

21. Kurkjian C, Kummar S (2009) Advances in the treatment of metastatic colorectal cancer. Am J Ther 16:412-420

22. Vilar E, Gruber SB (2010) Microsatellite instability in colorectal cancer-the stable evidence. Nat Rev Clin Oncol 7:153-162

23. Sinicrope FA, Sargent DJ (2012) Molecular pathways: microsatellite instability in colorectal cancer: prognostic, predictive, and therapeutic implications. Clin Cancer Res 18:1506-1512

24. Han JY, Lim HS, Shin ES et al (2006) Comprehensive analysis of UGT1A polymorphisms predictive for pharmacokinetics and treatment outcome in patients with non-small-cell lung cancer treated with irinotecan and cisplatin. J Clin Oncol 24:2237-2244

25. Kaniwa N, Kurose K, Jinno H et al (2005) Racial variability in haplotype frequencies of UGT1A1 and glucuronidation activity of a novel single nucleotide polymorphism $686 \mathrm{C}>\mathrm{T}$ (P229L) found in an African-American. Drug Metab Dispos 33:458-465

26. Saeki M, Saito Y, Jinno H et al (2006) Haplotype structures of the UGT1A gene complex in a Japanese population. Pharmacogenomics $\mathrm{J}$ 6:63-75

27. Vasen HF, Moslein G, Alonso A et al (2007) Guidelines for the clinical management of Lynch syndrome (hereditary non-polyposis cancer). J Med Genet 44:353-362

28. Abbott DE, Cantor SB, Rodriguez-Bigas MA, Chang GJ, Lynch PM, Feig BW, Skibber JM, You YN, University of Texas M. D. Anderson Cancer Center, Houston, TX (2012) Detecting hereditary nonpolyposis colorectal cancer syndrome (HNPCC) in patients with colorectal cancer (CRC): optimal strategies at lower costs. J Clin Oncol 30:(suppl 4; abstr 396)

29. Hampel H, Frankel WL, Martin E et al (2005) Screening for the Lynch syndrome (hereditary nonpolyposis colorectal cancer). N Engl J Med 352:1851-1860

30. Taylor CF, Charlton RS, Burn J et al (2003) Genomic deletions in MSH2 or MLH1 are a frequent cause of hereditary non-polyposis colorectal cancer: identification of novel and recurrent deletions by MLPA. Hum Mutat 22:428-433

31. van der Klift H, Wijnen J, Wagner A et al (2005) Molecular characterization of the spectrum of genomic deletions in the mismatch repair genes MSH2, MLH1, MSH6, and PMS2 responsible for hereditary nonpolyposis colorectal cancer (HNPCC). Genes Chromosomes Cancer 44:123-138

32. Grabowski M, Mueller-Koch Y, Grasbon-Frodl E et al (2005) Deletions account for $17 \%$ of pathogenic germline alterations in MLH1 and MSH2 in hereditary nonpolyposis colorectal cancer (HNPCC) families. Genet Test 9:138-146

33. Perez-Cabornero L, Velasco E, Infante $M$ et al (2009) A new strategy to screen MMR genes in Lynch syndrome: HA-CAE, MLPA and RT-PCR. Eur J Cancer 45:1485-1493

34. Wang Y, Friedl W, Lamberti C et al (2003) Hereditary nonpolyposis colorectal cancer: frequent occurrence of large genomic deletions in MSH2 and MLH1 genes. Int J Cancer 103:636-641

35. Wijnen J, van der Klift $\mathrm{H}$, Vasen $\mathrm{H}$ et al (1998) MSH2 genomic deletions are a frequent cause of HNPCC. Nat Genet 20:326-328

36. Peltomaki P (2005) Lynch syndrome genes. Fam Cancer 4:227-232

37. Moelans CB, Verschuur-Maes AH, van Diest PJ (2011) Frequent promoter hypermethylation of BRCA2, CDH13, MSH6, PAX5,
PAX6 and WT1 in ductal carcinoma in situ and invasive breast cancer. J Pathol 225:222-231

38. Wang Y, Friedl W, Sengteller M et al (2002) A modified multiplex PCR assay for detection of large deletions in MSH2 and MLH1. Hum Mutat 19:279-286

39. Trepanier A, Ahrens M, McKinnon W et al (2004) Genetic cancer risk assessment and counseling: recommendations of the national society of genetic counselors. J Genet Couns 13:83-114

40. Resta R, Biesecker BB, Bennett RL et al (2006) A new definition of Genetic Counseling: National Society of Genetic Counselors' Task Force report. J Genet Couns 15:77-83

41. Lindor NM, Petersen GM, Hadley DW et al (2006) Recommendations for the care of individuals with an inherited predisposition to Lynch syndrome: a systematic review. JAMA 296:1507-1517

42. Baglietto L, Lindor NM, Dowty JG et al (2010) Risks of Lynch syndrome cancers for MSH6 mutation carriers. J Natl Cancer Inst 102:193-201

43. Hendriks YM, Wagner A, Morreau H et al (2004) Cancer risk in hereditary nonpolyposis colorectal cancer due to MSH6 mutations: impact on counseling and surveillance. Gastroenterology 127:17-25

44. Kolodner RD, Tytell JD, Schmeits JL et al (1999) Germ-line msh6 mutations in colorectal cancer families. Cancer Res 59:5068-5074

45. Wagner A, Hendriks Y, Meijers-Heijboer EJ et al (2001) Atypical HNPCC owing to MSH6 germline mutations: analysis of a large Dutch pedigree. J Med Genet 38:318-322

46. Win AK, Young JP, Lindor NM et al (2012) Colorectal and other cancer risks for carriers and noncarriers from families with a DNA mismatch repair gene mutation: a prospective cohort study. J Clin Oncol 30:958-964

47. Box JC, Rodriguez-Bigas MA, Weber TK et al (1999) Clinical implications of multiple colorectal carcinomas in hereditary nonpolyposis colorectal carcinoma. Dis Colon Rectum 42:717-721

48. Lynch HT, Smyrk TC, Watson P et al (1993) Genetics, natural history, tumor spectrum, and pathology of hereditary nonpolyposis colorectal cancer: an updated review. Gastroenterology 104:1535-1549

49. Mecklin JP, Jarvinen HJ (1986) Clinical features of colorectal carcinoma in cancer family syndrome. Dis Colon Rectum 29:160-164

50. Watson P, Lin KM, Rodriguez-Bigas MA et al (1998) Colorectal carcinoma survival among hereditary nonpolyposis colorectal carcinoma family members. Cancer 83:259-266

51. Niessen RC, Kleibeuker JH, Westers H et al (2009) PMS2 involvement in patients suspected of Lynch syndrome. Genes Chromosomes Cancer 48:322-329

52. Peltomaki P (2003) Role of DNA mismatch repair defects in the pathogenesis of human cancer. J Clin Oncol 21:1174-1179

53. Desai TK, Barkel D (2008) Syndromic colon cancer: Lynch syndrome and familial adenomatous polyposis. Gastroenterol Clin N Am 37:47-72, vi

54. Acharya S, Wilson T, Gradia S et al (1996) hMSH2 forms specific mispair-binding complexes with hMSH3 and hMSH6. Proc Natl Acad Sci USA 93:13629-13634

55. Boland CR, Goel A (2010) Microsatellite instability in colorectal cancer. Gastroenterology 138(2073-2087):e2073

56. Poynter JN, Siegmund KD, Weisenberger DJ et al (2008) Molecular characterization of MSI-H colorectal cancer by MLHI promoter methylation, immunohistochemistry, and mismatch repair germline mutation screening. Cancer Epidemiol Biomarkers Prev 17:3208-3215

57. Samowitz WS, Curtin K, Ma KN et al (2001) Microsatellite instability in sporadic colon cancer is associated with an improved prognosis at the population level. Cancer Epidemiol Biomarkers Prev 10:917-923 